\title{
Balancing Cognitive and Motivational Scaffolding in Tutorial Dialogue
}

\author{
Kristy Elizabeth Boyer ${ }^{\mathrm{a}}$, Robert Phillips ${ }^{\mathrm{ab}}$, Michael Wallis ${ }^{\mathrm{ab}}$, \\ Mladen Vouk ${ }^{\mathrm{a}}$, and James Lester ${ }^{\mathrm{a}}$ \\ ${ }^{\mathrm{a} D e p a r t m e n t ~ o f ~ C o m p u t e r ~ S c i e n c e, ~ N o r t h ~ C a r o l i n a ~ S t a t e ~ U n i v e r s i t y ~}$ \\ ${ }^{\mathrm{b}}$ Applied Research Associates, Inc. \\ Raleigh, North Carolina, USA \\ \{keboyer, rphilli, mdwallis, vouk, lester\}@ncsu.edu
}

\begin{abstract}
A key challenge in the design of tutorial dialogue systems is identifying tutorial strategies that can effectively balance the tradeoffs between cognitive and affective student outcomes. This balance is problematic because the precise nature of the interdependence between cognitive and affective strategies is not well understood. Furthermore, previous studies suggest that some cognitive and motivational goals are at odds with one another because a tutorial strategy designed to maximize one may negatively impact the other. This paper reports on a tutorial dialogue study that investigates motivational strategies and cognitive feedback. It was found that the choice of corrective tutorial strategy makes a significant difference in the outcomes of both student learning gains and self-efficacy gains.
\end{abstract}

\section{Introduction}

Recent years have seen the emergence of a broader view of learning as a complex process involving both cognitive and affective states. To empirically explore these issues, a number of intelligent tutoring systems (ITSs) (e.g., AutoTutor [1], Betty's Brain [2], ITSpoke [3], M-Ecolab [4], and MORE [5]) are being used as platforms to investigate the impact of tutorial interactions on affective and motivational outcomes (e.g., self-efficacy) along with purely cognitive measures (i.e., learning gains). A central problem in this line of investigation is identifying tutorial strategies (e.g., [6]) that can appropriately balance the tradeoffs between cognitive and affective student outcomes [7]. While a rich set of cognitive and affective tutorial strategies is emerging (e.g., [8]), the precise nature of the interdependence between these types of strategies is not well understood. The extent to which each type of strategy, and specific instances of it in certain contexts, may be used to enhance tutorial effectiveness is an important question to designers of ITSs.

This paper reports on an empirical study to compare the impact of certain cognitive and motivational tutorial strategies on student learning and self-efficacy in humanhuman tutoring. Specifically, we consider the motivational strategies of praise and 
reassurance [7] and the category of informational tutorial utterances termed cognitive feedback [2, 8]. Following the approach of Forbes-Riley and colleagues [3, 9], utterances from a corpus of human-human tutorial dialogues are annotated with dialogue acts. Then, adopting the approach proposed by Ohlsson et al. [10], statistical modeling techniques are employed to quantify the relative impact of these different tutorial strategies on the outcomes of interest (in this case, learning and self-efficacy gains). By mining a corpus of human-human tutorial dialogues for naturallyoccurring corrective strategies (i.e., tutorial moves in response to plausibly incorrect student problem-solving actions), we induce tutorial dialogue strategies that embody the regularities of effective tutorial dialogue across multiple tutoring sessions.

A key finding of the study is that the choice of corrective tutorial strategy has a significant impact on both the learning gains and the self-efficacy gains of students. The results reinforce related findings (e.g., $[2,7,11])$ that suggest some cognitive and motivational goals are at odds with one other because a tutorial strategy designed to maximize one set of goals (e.g., cognitive goals) can negatively impact the other. However, the study reported here also found that a strategy that provides students with positive cognitive feedback as a corrective approach can strike a "delicate balance" [1] and achieve desirable motivational and cognitive outcomes.

\section{Related Work}

Much of the research on motivation conducted in the ITS community is theoretically grounded in frameworks developed in the cognitive science community over the past two decades (e.g., [12, 13, 14]). Chief among these results is Keller's theory that student motivation plays a key role in the learning process [12]. This view is seconded by Lepper [7], who states that the most effective tutors give equal attention to both the motivational and cognitive concerns of students. Lepper et al. [7] refine Keller's model by postulating that motivation is comprised of confidence, challenge, control, and curiosity. Lepper [7] further identifies the two strategies of praise and reassurance as direct means of bolstering student confidence. These strategies are a form of "verbal persuasion," also identified by Bandura [15], as one way of increasing self-efficacy.

An increasingly active area of investigation is the search for tutorial strategies that address the complementary cognitive and affective concerns that shape the tutoring process [16]. Porayska-Pomsta and Pain [8] use dialogue analysis to classify cognitive and affective feedback ${ }^{1}$ in terms of the degree to which each addresses a student's need for both autonomy and approval. Forbes-Riley and Litman (e.g., [18]) employ bigram analysis at the dialogue act level to extract tutorial strategies for responding to student uncertainty. Corpus analysis techniques have also informed work by Marineau et al. [9] on the classification of tutorial acts, as well as work by Rosé et al. (e.g., [19]) and Ohlsson et al. [10] on modeling the effectiveness of tutorial strategies.

${ }^{1}$ We use feedback to refer to "information communicated to the learner that is intended to modify the learner's thinking or behavior for the purpose of improving learning" [17]. 
Developing a clear understanding of the tradeoffs between cognitive and affective feedback is an important next step in tutorial dialogue research. Prior investigations of tutorial feedback have established a foundational understanding of cognitive feedback in terms of how and when it is delivered (e.g., [20]). Jackson and Graesser [1] found the presence of cognitive feedback, as opposed to motivational "progress" feedback, was responsible for higher learning gains in experimental versions of AutoTutor; on the other hand, the presence of cognitive feedback lowered students' motivational ratings. A consistent finding observed by Tan and Biswas [2] was that students working with modified versions of Betty's Brain were able to learn better when given cognitive rather than affective feedback. Kelly and Weibelzahl [21] investigated a motivational strategy in which a student was progressively shown more of a hidden image after each successful step through the learning task. Students in the motivational treatment group showed larger increases in confidence levels compared with those in the control group, while there was no significant difference in learning gain. Finally, Wang et al. [22] found that tutors who gave polite feedback facilitated higher student self-efficacy gains, while learning was nearly unaffected.

Beyond these broadly observable tradeoffs, investigators have also found that tutorial strategies may impact student subgroups (e.g., low ability vs. high ability students) in different ways. Rebolledo-Mendez et al. [4] explored the effect of enhancing a tutoring system with motivational scaffolding. In M-Ecolab, initially unmotivated students were found to perform better with motivational adaptation and feedback, while students who were already motivated did not benefit from the motivational support. In a study of perceived politeness (a motivational aspect of tutorial utterances), Mayer et al. [23] found students who were experienced with computers were less bothered by direct commands from a machine, while inexperienced students were more apt to appreciate politeness.

\section{Corpus Study}

To determine the effect of specific tutorial strategies on learning and self-efficacy, a human-human tutoring corpus study was conducted. With a focus on tutorial strategies for addressing questionable student problem-solving actions, the study investigated student-tutor interactions in the domain of introductory computer science. The corpus consists of three types of events: tutor utterances, student utterances, and student problem-solving actions (in this case, programming events in which students create statements in Java programs).

\subsection{Experimental Design}

Subjects. Forty-three volunteers from a university-level introductory computer programming class attended a single tutoring session each. Subjects were not compensated for their participation; the indirect reward was that participants were not required to attend their weekly computer science laboratory class because they fulfilled the attendance requirement through study participation. 
Procedure. At the beginning of each tutoring session, subjects completed a questionnaire containing items designed to gauge self-efficacy as it relates to completing the computer science task [24]. Subjects also completed a pre-test that measured conceptual knowledge related to the learning task. The tutor and student were in separate rooms during the 55-minute tutoring session, working through an extended version of a software package developed to facilitate remote collaborative programming [25]. This software allowed tutors to observe student problem-solving actions in real time while carrying on conversations through a textual dialogue interface. Students were not aware of any tutor characteristics (e.g., name, gender). Upon completion of the session, students filled out a post-questionnaire and a posttest containing questions that were analogues to the pre-tutoring versions.

Tutors. Fourteen volunteer tutors were paired blindly with students based solely on scheduling availability. Tutors were in separate rooms from students and were not made aware of any student characteristics (e.g., self-efficacy rating, gender, pre-test score). All tutors were themselves students in a Department of Computer Science. Two were advanced undergraduates, and the remaining twelve were graduate students. All fourteen tutors reported experience as a peer tutor, and ten tutors had also served as teaching assistants in a university computing course for one or more semesters Of these ten, three had also served as primary instructors in a universitylevel introductory programming course. Four of the tutors were female. While these and other tutor characteristics may be useful for predicting what tutors will do (and perhaps even why), this paper begins with what tutors did do and goes on to draw conclusions on the relative effectiveness of various strategies [10].

\subsection{Corpus Characteristics}

The raw corpus contains 4,864 dialogue moves: 1,528 student utterances and 3,336 tutor utterances. As a chronology of tutorial dialogue interleaved with student problem-solving (programming) actions that took place during the tutoring sessions, the corpus contains 29,996 programming keystrokes and 1,277 periods of scrolling all performed by students. Other problem-solving actions, such as opening and closing files or running the program, were sparse and were therefore omitted here.

Of the 3,336 tutor utterances, 1,243 occur directly after "questionable" student problem-solving action. (The notion of "questionable" is defined below.) This subset of tutorial utterances serves as the basis for the tutorial strategy comparison.

\subsection{Problem-Solving Act Tagging}

Student problem-solving actions were logged throughout the tutoring sessions. The two actions under consideration for this analysis are: typing in the programming interface and scrolling in the program editor window. To interpret the raw logged student problem-solving actions, these events were automatically tagged using a heuristic measure for correctness. This heuristic represents just a first step toward automatically classifying student actions in the problem-solving environment: if a 
programming keystroke (character) survived until the end of the session, this event was tagged promising. This heuristic is based on the observation that the subtasks in this learning task were accomplished in a linear fashion, with tutors not allowing students to move forward until the previously implemented steps were judged to be correct. Conversely, if a programming keystroke (character) did not survive until the end of the session, the problem-solving act was tagged questionable. The rationale for this rule is that non-surviving characters were subsequently displaced or removed for some reason, meaning they were plausibly incorrect to start with. Finally, periods of consecutive scrolling were marked questionable because in this context, scrolling was almost uniformly undertaken by a student who was confused and looking for answers in irrelevant task scaffolding.

\subsection{Dialogue Act Annotation}

Because utterances communicate through two orthogonal channels, a cognitive channel and a motivational channel, each utterance was annotated with both a cognitive dialogue tag and a motivational dialogue tag. The dialogue act tag set, which consists of sixteen cognitive acts plus six motivational/affective acts, is an extension of the tag set presented in [26]. Table 1 displays the subset of this dialogue act tagging scheme relevant to the current study.

The entire corpus was tagged by a single human annotator, with a second tagger marking 1,418 of the original 4,864 utterances. The resulting kappa statistics were 0.76 in the cognitive channel and 0.64 in the motivational/affect channel. ${ }^{2}$

\section{Analysis and Results}

Overall, the tutoring sessions were effective: they yielded learning gains (mean 5.9\%, median $7.9 \%$ ), which were statistically significant $(p=0.038)$, and they produced selfefficacy gains (mean $12.1 \%$, median $12.5 \%$ ), which were also statistically significant $(p<0.0001)$.

Analyses revealed that statistically significant relationships hold between tutorial strategy and learning, as well as between tutorial strategy and self-efficacy gains. First, the values of learning gain and self-efficacy gain were grouped into binary categories ("Low", "High") based on the median value. Multiple logistic regression was then applied with the gain category as the predicted value and tutorial strategy, incoming self-efficacy rating, and pre-test score as predictors. ${ }^{3}$ Multiple logistic regression was chosen over multiple linear regression because the learning instruments (10 items each) yielded few distinct values of learning gain. Logistic regression computes the odds of a particular outcome over another (e.g., "Having high

\footnotetext{
${ }^{2}$ This kappa was computed on all student and tutor utterances using the full tagging scheme.

3 To control for the possibility that student outcomes were predicted entirely by incoming student characteristics rather than by any tutorial action, pre-test score and incoming selfefficacy rating were treated as predictors in all models.
} 
Table 1: Relevant Tutorial Dialogue Acts

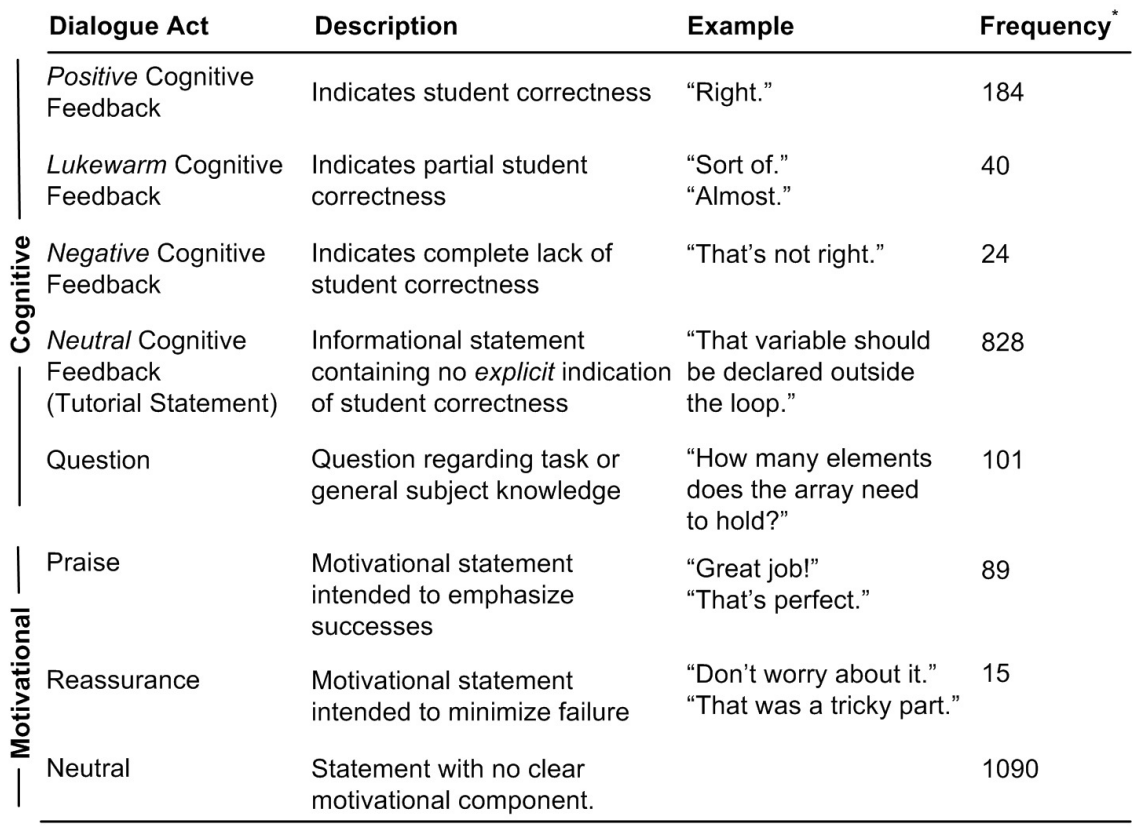

* The frequency column indicates a simple frequency out of 1,243 tutor utterances that followed questionable student problem-solving actions in the corpus. These dialogue acts are not mutually exclusive and are not exhaustive since this table displays only a subset of all dialogue acts.

learning gain versus low learning gain") given one value of the predictor variable over another (e.g., "The tutorial strategy was positive feedback instead of praise").

\subsection{Presence of Tutorial Encouragement}

We first consider two categories of corrective tutorial utterances: those with and those without explicit encouragement (i.e., praise or reassurance). Both these categories may, but need not, contain cognitive feedback components. (We restrict the analysis to only cognitive feedback in the next subsection, and later omit all such feedback to consider standalone tutorial encouragement.) A logistic regression model quantified the significant relationships between tutorial encouragement and learning gain, revealing that after accounting for the effects of pre-test score and incoming selfefficacy rating (both of which were significant in the model with $p<0.001$ ), observations containing tutorial encouragement were $56 \%{ }^{4}$ less likely to result in high learning gain than observations without explicit tutorial encouragement $(p=0.001)$.

4 This value and its counterparts throughout the paper represent logistic regression point estimates of odds ratio (analogous to the regression coefficient in multiple linear regression). The accompanying $p$-value indicates the level at which the predictor variable was significant in the model. 
On the other hand, tutorial encouragement was weakly linked to self-efficacy gains, with explicit encouragement being 57\% more likely to result in high self-efficacy gain than tutorial responses that had no explicit praise or reassurance $(p=0.054)$. These models suggest that the presence of tutorial encouragement in response to questionable student problem-solving action is weakly linked to self-efficacy gain but may detract from learning gain.

\subsection{Adding Encouragement to Cognitive Feedback}

We now consider only corrective tutorial acts that were tagged as cognitive feedback and compare the relative impact of those with and without explicit tutorial praise or reassurance. Because the co-occurrence of cognitive feedback with reassurance was very low $(n=2)$, we omit this strategy from consideration and compare the two strategies of purely cognitive feedback and cognitive feedback plus praise. A logistic regression model built as described above revealed that observations in which the tutor used cognitive feedback plus praise were associated with $40 \%$ lower likelihood of high learning gain than observations in which the tutor used purely cognitive feedback. No impact was observed on self-efficacy gain. These results suggest that in response to questionable student problem-solving action, to achieve learning gains, purely cognitive feedback is preferred over cognitive feedback plus praise, while selfefficacy gain does not appear to be impacted either way.

\subsection{Standalone Tutorial Encouragement}

In this corpus, tutorial encouragement is sometimes encountered with no cognitive feedback component; that is, the tutorial utterance is in no way aimed at giving substantive task-related feedback, but instead, is aimed at the student's motivational or affective state through explicit praise or reassurance. We now consider this tutorial strategy of standalone motivational acts. Unlike the previous results that had a consistent (or no statistically significant) impact on student sub-groups and were therefore reported only for the general student population, purely motivational statements appear to affect low and high self-efficacy students differently. A separate logistic regression was run for the low initial self-efficacy and high initial selfefficacy student groups. Among students with low incoming self-efficacy, observations in which the tutor employed a standalone motivational act were $300 \%$ as likely to be in the high self-efficacy gain group as observations in which the tutor employed a purely cognitive statement or a cognitive statement combined with encouragement $(p=0.039)$. In contrast, among students with high initial self-efficacy, a purely motivational tactic resulted in $90 \%$ lower odds of being in the high selfefficacy gain group. Standalone motivational acts showed no statistically different impact on learning gain compared to other tutorial acts $(p=0.268)$. This relationship held for both the low self-efficacy $(p=0.216)$ and high self-efficacy subgroups $(p=0.441)$ with regard to impact on learning gain. These results suggest that standalone praise or reassurance may be useful for increasing self-efficacy gain among low initial self-efficacy students, but may decrease self-efficacy gain in high 
initial self-efficacy students. In addition, standalone praise or reassurance does not appear helpful for learning gains.

\subsection{Superiority of Positive Cognitive Feedback}

We have seen evidence thus far that explicit tutor encouragement in the form of praise or reassurance has mixed effects on learning and self-efficacy gains. We now consider the class of purely cognitive tutorial moves, i.e., all tutorial acts that have no explicit encouragement attached. As presented in Section 3.4, the strategies under consideration here are positive, lukewarm, negative, and neutral cognitive feedback plus tutorial questions. Because positive cognitive feedback related similarly to each of the other types of cognitive moves, we forego pairwise comparisons and instead contrast positive cognitive feedback against the group of all other purely cognitive strategies. Chi-square analysis reveals positive cognitive feedback had a significantly different impact on self-efficacy than other strategies $(p=0.0028)$. A logistic regression refined the relationship, revealing positive feedback resulted in $190 \%$ increased odds of high student self-efficacy gain compared to the other cognitive strategies $(p=0.0057)$. Positive cognitive feedback did not differ significantly from other types of cognitive strategies in a Chi-square comparison with respect to learning gains $(p=0.390)$. The models thus suggest when dealing with questionable student problem-solving action, positive cognitive feedback is preferable to other types of cognitive feedback for eliciting self-efficacy gains, but this type of feedback is not found to be better or worse than other cognitive feedback for effecting learning gains.

\section{Discussion}

The study found that the presence of direct tutorial praise or encouragement in response to questionable student problem-solving action increased the odds that the student exhibited high self-efficacy gain, while lowering the odds of high learning gain. The study also found that purely cognitive feedback was preferable for learning gains compared to cognitive feedback with an explicitly motivational component. These empirical findings are consistent with theories of Lepper [7] who observed that some cognitive and affective goals in tutoring are "at odds." The results also echo quantitative results from other domains such as qualitative physics [1] and river ecosystems [2] that, in general, overt motivational feedback contributes to motivation but cognitive feedback matters more for learning.

In this study, standalone motivational utterances in response to questionable problem-solving action increased the likelihood of high self-efficacy gain among low incoming self-efficacy students. This motivational tactic, however, reduced the likelihood of self-efficacy gain in students with initially high self-efficacy. These results confirm empirical findings from the domain of ecology [4] in which only unmotivated students benefited from extra motivational scaffolding. While it is true that students with initially high confidence have less room for self-efficacy gain in the first place, it is also likely the case that students with high confidence or high ability may be less prone to need or appreciate motivational tactics such as politeness [23]. 
Of the tutorial strategies that occurred in the corpus, positive cognitive feedback emerged as an attractive approach for responding to plausibly incorrect student problem-solving actions. Responding positively (e.g., "Right") to questionable student actions is an example of indirect correction, which is recognized as a polite strategy (e.g., $[8,22]$ ). As such, the positive feedback approach seems to have an implicit, yet perceptible, motivational component while retaining its usefulness as cognitive feedback. Qualitative inspection of the corpus indicates positive cognitive feedback in response to plausibly incorrect problem-solving actions was usually followed by neutral cognitive feedback that served to more informatively, yet indirectly, point out student errors. Thus the benefits of positive cognitive feedback may also indicate (by proxy) the effectiveness of Lepper's indirect feedback acts [7].

\section{Conclusions and Future Work}

Balancing cognitive and motivational scaffolding has emerged as a key problem in tutorial dialogue. To investigate these issues, a corpus study of naturalistic humanhuman tutorial dialogue in the domain of computer science was conducted to determine the most effective use of motivation in the context of feedback for problematic student actions. The results suggest that positive cognitive feedback may prove an appropriate strategy for responding to questionable student problem-solving action in task-oriented tutorial situations because of its potential for addressing the sometimes competing cognitive and affective needs of students. For low self-efficacy students, however, it was found that direct standalone encouragement can be used to bolster self-efficacy, but the same standalone encouragement may not be helpful for high self-efficacy students.

In this work we have considered a limited set of motivational dialogue acts, namely praise and reassurance. Important future work will target an expanded set of affective dialogue acts to facilitate continued exploration of motivational and affective phenomena in this context. Also important will be expanding the window of consideration to pairs that include both student and tutor utterances, along with tuples that include three acts or more (e.g., problem-solving action, tutor utterance, student utterance) to model the effects of higher-level tutorial strategies. Finally, the current results reflect human-human tutoring strategies that proved to be effective; however, it remains to be seen whether these same strategies can be successfully employed in tutorial dialogue systems. Continuing to identify and empirically compare the effectiveness of alternative tutorial strategies will build a solid foundation for choosing tutorial strategies that balance the cognitive and affective concerns surrounding the complex processes of teaching and learning through tutoring.

Acknowledgments. The authors wish to thank Scott McQuiggan and the members of the Intellimedia Center for Intelligent Systems for their ongoing intellectual contributions, and the Realsearch Group at NC State University for extensive project development support. This work was supported in part by the National Science Foundation through Grant REC-0632450, an NSF Graduate Research Fellowship, and the STARS Alliance Grant CNS-0540523. Any opinions, findings, conclusions or 
recommendations expressed in this material are those of the author(s) and do not necessarily reflect the views of the National Science Foundation. Support was also provided by North Carolina State University through the Department of Computer Science and the Office of the Dean of the College of Engineering.

\section{References}

[1] Jackson, G. T., Graesser, A. C. Content matters: An investigation of feedback categories within an ITS. In Luckin, R., Koedinger, K. R., Greer, J. (Eds.), Proceedings of AIED 2007, vol. 158, pp. 127-134, IOS Press (2007)

[2] Tan, J., Biswas, G. The role of feedback in preparation for future learning: A case study in learning by teaching environments. In Ikeda, M., Ashley, K., Chan, T.-W. (Eds.), Proceedings of ITS 2006. LNCS, vol. 4053, pp. 370-381, Springer-Verlag Berlin / Heidelberg (2006)

[3] Forbes-Riley, K., Litman, D., Huettner, A., Ward, A. Dialogue-learning correlations in spoken dialogue tutoring. In Looi, C-K., Mccalla, G., Bredeweg, B., Breuker, J. (Eds.), Proceedings of AIED, pp. 225-232, IOS Press (2005)

[4] Rebolledo-Mendez, G., du Boulay, B., Luckin, R. Motivating the learner: An empirical evaluation. In Ikeda, M., Ashlay, K. D., Chan, T.-W. (Eds.), Proceedings of ITS 2006. LNCS, vol. 4053, pp. 545-554, Springer Verlag Berlin / Heidelberg (2006)

[5] del Soldato, T., du Boulay, B. Implementation of motivational tactics in tutoring systems. Journal of Artificial Intelligence in Education, 6(4), pp. 337-378, AACE (1995)

[6] Graesser, A. C., Person, N. K., Magliano., J. P. Collaborative dialogue patterns in naturalistic one-to-one tutoring. Applied Cognitive Psychology, 9(6), pp. 495-522, John Wiley \& Sons, Ltd (1995)

[7] Lepper, M. R., Woolverton, M., Mumme, D. L., Gurtner, J. Motivational techniques of expert human tutors: Lessons for the design of computer-based tutors. In Lajoie, S.P., Derry, S. J. (Eds.), Computers as Cognitive Tools, pp. 75-105, Lawrence Erlbaum Associates, Inc., Hillsdale NJ (1993)

[8] Porayska-Pomsta, K., Pain, H. Providing cognitive and affective scaffolding through teaching strategies: Applying linguistic politeness to the educational context. In J.C. Lester, Vicari, R. M., Paraguaçu, F. (Eds.), Proceedings of ITS 2004. LNCS vol. 3220, pp. 77-86, Springer-Verlag Berlin / Heidelberg (2004)

[9] Marineau, J., Wiemer-Hastings. P., Harter, D., Olde, B., Chipman, P., Karnavat A., Pomeroy, V., Rajan, S., Graesser, A., and the Tutoring Research Group. Classification of speech acts in tutorial dialog. Proceedings of the ITS 2000 Workshop on Modeling Human Teaching Tactics and Strategies, pp. 65-71, (2000)

[10] Ohlsson, S., Di Eugenio., B., Chow, B., Fossati, D., Lu, X., Kershaw., T.C. Beyond the code-and-count analysis of tutoring dialogues. In Luckin, R., Koedinger, K. R., Greer, J. (Eds.), Proceedings of AIED 2007, vol. 158, pp. 349-356, IOS Press (2007)

[11] Person, N. K., Kreuz, R. J., Zwaan, R. A., Graesser, A. C.: Pragmatics and pedagogy: Conversational rules and politeness strategies may inhibit effective tutoring. Cognition and Instruction, 13(2), pp. 161-188, Lawrence Erlbaum Associates, Inc., Hillsdale, NJ (1995)

[12] Keller, J.M.: Motivational design of instruction. In: Reigeluth, C.M. (Ed.): Instructional-Design Theories and Models: An Overview of Their Current Status, pp. 383-429, Lawrence Erlbaum Associates, Inc., Hillsdale, NJ (1983) 
[13] Cameron, J., Pierce, W. D.: Reinforcement, reward, and intrinsic motivation: A meta-analysis. Review of Educational Research, 64(3), pp. 363-423, American Educational Research Association, Washington, DC (1994)

[14] Deci, E. L., Koestner, R., Ryan, R. M.: Extrinsic rewards and intrinsic motivation in education: Reconsidered once again. Review of Educational Research, 71(1), pp. 127. American Educational Research Association, Washington, DC (2001)

[15] Bandura, A. Self-Efficacy: The Exercise of Control. W.H. Freeman and Company, New York (1997)

[16] Lehman, B., Matthews, M., D'Mello, S., Person, N. What are you feeling? Investigating student affective states during expert human tutoring sessions. To appear in Proceedings of ITS 2008, Springer-Verlag, Berlin/Heidelberg (in press)

[17] Shute, V.J. Focus on formative feedback. Technical Report RR-07-11, Educational Testing Service, Princeton, NJ (2007)

[18] Forbes-Riley, K., Litman, D. Using bigrams to identify relationships between student certainness states and tutor responses in a spoken dialogue corpus. Proceedings of the 6th SIGdial Workshop on Discourse and Dialogue, Lisbon, Portugal (2005)

[19] Rosé, C. P., Bhembe, D., Siler, S., Srivastava, R., VanLehn, K. The role of why questions in effective human tutoring. In Hoppe, U., Verdejo, F., Kay, J. (Eds.), Proceedings of AIED 2003, pp. 55-62, IOS Press (2003)

[20] Koedinger, K. R., Anderson, J. R., Hadley, W. H., Mark, M. A. Intelligent tutoring goes to school in the big city. International Journal of Artificial Intelligence in Education, vol. 8, pp. 30-43 (1997)

[21] Kelly, D., Weibelzahl, S. Raising confidence levels using motivational contingency design techniques. In Ikeda, M., Ashley, K., Chan, T.-W. (Eds.), Proceedings of ITS 2006. LNCS, vol. 4053, pp. 535-544, Springer-Verlag Berlin / Heidelberg (2006)

[22] Wang, N., Johnson, L., Rizzo, P., Shaw, E., Mayer, R.E. Experimental evaluation of polite interaction tactics for pedagogical agents. Proceedings of the 10th International Conference on Intelligent User Interfaces, pp. 12-19. San Diego, CA (2005)

[23] Mayer, R. E., Johnson, W. L., Shaw, E., Sandhu, S. Constructing computer-based tutors that are socially sensitive: Politeness in educational software. International Journal of Human-Computer Studies, 64(1), pp. 36-42, Academic Press, Inc., Duluth, MN (2006)

[24] Bandura, A. Guide for constructing self-efficacy scales. In Urdan, T., Pajares, F. (Eds.): Self-Efficacy Beliefs of Adolescents, pp. 307-337, Information Age Publishing, Greenwich, Connecticut (2006)

[25] Ho, C.-W., Raha, S., Gehringer, E., Williams, L. Sangam - A Distributed pair programming plug-in for eclipse. Proceedings of the 2004 OOPSLA Workshop on Eclipse Technology eXchange, pp. 73-77, Association for Computing Machinery Press, New York, NY, USA (2004)

[26] Boyer, K.E., Vouk, M.A., Lester, J.C. The influence of learner characteristics in task-oriented tutorial dialogue. In Luckin, R., Koedinger, K, Greer, J. (Eds.). Proceedings of AIED 2007, pp. 127-134, IOS Press (2007) 